\title{
CNS INFECTIONS
}

\section{SUBACUTE SCHIEROSING PANEANCEPHALITIS}

A 10-year old Nicaraguan boy with SSPE was treated with intraventricular biosynthetic alpha $2 \mathrm{~b}$ interferon (Schering, Intron A) 1 million units twice weekly for $5 \frac{1}{2}$ months, at the Children's Hospital of Los Angeles, Neurology Division, Los Angeles, CA. Measles history was unknown; CSF and serum measles titers were greater than 1:64 and 1:256,000, respectively. Fever developed for several hours and dystonic posturing worsened after each dose. The child remained in a vegetative state and neither clinical nor CSF indices improved with treatment. The authors question whether the choice of a biosynthetic form instead of the natural alpha interferon, used successfully in 3 patients previously (Panitch HS et al. Neurology 1986;36:562), might account for the lack of response in their patient. (Mitchell WF, Crawford TO. Intraventricular alpha $2 b$ interferon for SSPE. Neurology 1987; 37: 1884).

COMENT. The National SSPE Registry, now located at the Dept Neurology, Univ South Alabama College of Medicine, Mobile, Alabama, has recently begun to track results of treatment of SSPE (Dyken PR. Neurology $1987 ; 37: 1883)$. The use of the standard neurological disability scale as employed in the present study helps to eliminate bias in the evaluation of new therapies. The course of the disease is variable and the results of treatment in only a few cases are probably unreliable.

While on sabbatical at the Hospital for Sick Children, Great Ormond Street, London, 1986-87, I observed 6 cases of SSPE treated by intraventricular interferon, part of a large scale study with long-term results not yet evaluated. The treatment appeared to be arduous and poorly tolerated, sometimes interrupted by secondary infection, and stressful to the child, family, nurses, social workers as well as physicians involved. The EEG's were often worse after the initiation of therapy. Preliminary observations suggested that interferon might prolong the course of the disease but seemed to have no specific curative effect. An international collaborative study with standardized treatment protocol should be considered together with more effective WHO measles immunization programs to prevent this distressing disease.

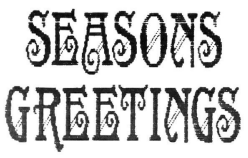

